\title{
REMARKS ON THE BOX PROBLEM
}

\author{
Nets Hawk Katz, Elliot Krop, and Mauro Maggioni
}

\section{Introduction}

In $[\mathrm{CCW}$, in connection with estimates on Fourier integral operators, the following question was raised. Let

$$
Q=[0,1]^{n} \subset \mathbb{R}^{n}
$$

be the unit cube in $n$ dimensions. Let $f$ be a $C^{n}$ function on $Q$ satisfying

$$
\frac{\partial^{n} f}{\partial x_{1} \partial x_{2} \ldots \partial x_{n}}>\Lambda
$$

What upper bound can be given on the measure of the set

$$
S=\{x \in Q:|f(x)|<1\} .
$$

One can attempt to bound the measure of the set using combinatorial means. By a simple application of the fundamental theorem of calculus - one sees there is constant $K_{n}$ depending only on the dimension so that for no sequence of pairs

$$
\left(x_{11}, x_{12}\right),\left(x_{21}, x_{22}\right), \ldots,\left(x_{n 1}, x_{n 2}\right)
$$

satisfying

$$
\Pi_{i=1}^{n}\left|x_{i 1}-x_{i 2}\right| \geq \frac{1}{K_{n} \Lambda},
$$

is it the case that the points $\left(x_{1 \alpha_{1}}, \ldots, x_{n \alpha_{n}}\right)$ are elements of $S$ for every choice of $\alpha_{1}, \ldots, \alpha_{n}$ running from 1 to 2 . Another way of saying this is that $S$ does not contain the corners of a large box with sides in the coordinate directions. We will say throughout this note that any set satisfying this restriction satisfies the (CCW)-condition with constants $\left(K_{n}, \Lambda\right)$.

A few applications of Cauchy Schwarz show that a set satisfying the (CCW) condition has measure at most $O\left(\Lambda^{\frac{1}{2^{n-1}}-\epsilon}\right)$ for any $\epsilon>0$. On the other hand,

Received November 21, 2000.

2000 Mathematics Subject Classification. Primary 35A07, Secondary 53B05.

The first author was partially supported by a National Science Foundation grant. 
this bound may not be sharp - no one knows how to find a (CCW) set that large. A very easy example of a $(\mathrm{CCW})$ set is as follows, however. Let

$$
f=\frac{\Lambda}{n !}\left(x_{1}+x_{2}+\ldots x_{n}-\frac{n}{2}\right)^{N} .
$$

The $N$ th mixed partial of $f$ is $\Lambda$. Let

$$
S=\left\{\left(x_{1}, \ldots, x_{n}\right):\left|f\left(x_{1}, \ldots, x_{n}\right)\right| \leq 1 .\right\}
$$

Clearly $S$ is the set of points $O\left(\Lambda^{\frac{1}{n}}\right)$ away from a plane which cuts the cube generically. Thus clearly $|S|=O\left(\Lambda^{\frac{1}{n}}\right)$. This index was observed in [CCW].

Thus we have an upper bound a little worse than $O\left(\Lambda^{\frac{1}{2^{n-1}}}\right)$ and a lower bound of $O\left(\Lambda^{\frac{1}{n}}\right)$. However $\frac{1}{2^{n-1}}$ becomes a lot smaller than $\frac{1}{n}$ as $n$ grows bigger than 2. The first interesting case is $n=3$ where the former is $\frac{1}{4}$ and the latter $\frac{1}{3}$.

One can make discrete analogues of this question. Let $I_{i j k}$ be an $N \times N \times N$ tensor of 1's and 0's. We say $I$ satisfies the (E) condition provided it does not contain 1's at all the corners of any nontrivial box. That is, $I$ satisfies the (E) condition, if for any $i_{1} \neq i_{2}, j_{1} \neq j_{2}, k_{1} \neq k_{2}$, we have that

$$
\Pi_{l=1}^{2} \Pi_{m=1}^{2} \Pi_{n=1}^{2} I_{i_{l} j_{m} k_{n}}=0 .
$$

We can ask: what is the best upper bound that can be proven for the number of 1's in any tensor $I$ satisfying the (E) condition? We refer to this as the box problem. It was first stated by Erdös [E] in the context of hypergraphs. We are interested in it mainly because of its relation to the work in [CCW].

A few applications of the Cauchy Schwarz inequality show that the number of 1 's is at most $O\left(N^{\frac{11}{4}}\right)$. Erdös [E] conjectured it to be sharp and it is the analogue of the $\frac{1}{4}$ above. One might ask - does one have an analogue of the lower bound with $\frac{1}{3}$ ? Simple numerology shows that this should consist of an example with $O\left(N^{\frac{8}{3}}\right)$ many 1's. Unfortunately, the best published example seems to have only $O\left(N^{\frac{13}{5}}\right)$ many 1's, see ([GRS], Lemma 2.4). This state of affairs is bad - since it would seem to indicate that such examples are far from providing new results on sets with the (CCW) condition. The [GRS] construction is inefficient in that it uses probabilistic methods. In its defense, one should note that in 5 and higher dimensions ([GRS], Lemma 2.5), it starts to beat the (CCW) index (and ties it in 4 dimensions) and it generally provides evidence for Erdös' conjecture.

We try to rectify the three dimensional situation.

Theorem. Let $p$ be any prime. There is a $p^{3}-1 \times p^{3}-1 \times p^{3}-1$ tensor I satisfying the $(E)$ condition containing $p^{2}\left(p^{3}-1\right)^{2}$ many 1 's.

This does not provide new results about sets with the (CCW) condition but any improvement in the index would.

We prove the theorem in Section 1. 


\section{Proof of Theorem}

We begin by recalling the standard construction of a sharp example for the two dimensional problem. It is simply part of the finite projective plane.

We consider the finite field $F_{q}$ with $q$ elements. (It must be that $q=p^{n}$ for some prime $p$ and some integer $n$.) We define a $q^{2}-1 \times q^{2}-1$ matrix $I_{i j}$ consisting of 1's and 0 's so that for no $i_{1} \neq i_{2}$ and $j_{1} \neq j_{2}$ is it the case that

$$
I_{i_{1} j_{1}} I_{i_{1} j_{2}} I_{i_{2} j_{1}} I_{i_{2} j_{2}}=1
$$

We shall index the $q^{2}-1$ rows $i$ by $F_{q}^{2} \backslash\{(0,0)\}$, which is the plane over $F_{q}$ excluding the origin. We shall index the columns $j$ by the set of lines in $F_{q}^{2}$ not containing the origin. There are $q^{2}-1$ different such lines, given by the equations

$$
a x+b y=1,
$$

where $(a, b) \in F_{q}^{2} \backslash\{(0,0)\}$. (Thus we notice a duality between the set of lines we consider and the set of points we consider.) Now we define $I_{i j}=1$ if the point indexed by $i$ is contained in the line indexed by $j$. Now since the intersection between two different lines is at most a point, we cannot have (1) whenever $j_{1} \neq j_{2}$ and $i_{1} \neq i_{2}$. We produce such an $I$ with $q\left(q^{2}-1\right)$ many 1 's, which is close to the most possible.

We now attempt to generalize this idea to three dimensions in order to prove the theorem. We shall fix a prime $p$ and do geometry in $F_{p}^{3} \backslash\{0\}$. A plane in $F_{p}^{3}$ is an equation of the form

$$
a x+b y+c z=1,
$$

with

$$
(a, b, c) \in F_{p}^{3} \backslash\{0\} .
$$

Notice that here we have point-plane duality. Occasionally, we shall consider $F_{p}^{3}$ to be identified with $F_{p^{3}}$ which is the extension of $F_{p}$ by an irreducible cubic. We let $r$ be a root of this cubic. Then an element of $F_{p^{3}}$ can be written uniquely as

$$
\alpha=a+r b+r^{2} c
$$

with $a, b, c \in F_{p}$. We say $\alpha$ is real if $b=c=0$. Otherwise, we say $\alpha$ is complex.

We are now ready to define our construction of $I_{i j k}$ a $\left(p^{3}-1\right) \times\left(p^{3}-1\right) \times$ $\left(p^{3}-1\right)$ tensor of 1's and 0's. We index $i$ and $j$ by $F_{p^{3}} \backslash\{0\}$ and we index $k$ by $F_{p}^{3} \backslash\{(0,0,0)\}$. We will denote multiplication in $F_{p^{3}}$ by *. To each element $\alpha \in F_{p^{3}} \backslash\{0\}$, we assign a plane $P_{\alpha}$ in $F_{p}^{3} \backslash\{(0,0,0)\}$ by saying that $P_{\alpha}$ is given by the equation

$$
a x+b y+c z=0
$$

when

$$
\alpha=a+r b+r^{2} c .
$$

Now we define $I_{i j k}$. We let $I_{i j k}=1$ if $k \in P_{i * j}$, and we let $I_{i j k}=0$ otherwise. 
Clearly $I_{i j k}$ has $p^{2}\left(p^{3}-1\right)^{2}$ many 1 's. To prove the theorem, it now suffices to show that $I$ satisfies the (E)-condition. We need only show that if $i_{1} \neq i_{2}$ and $j_{1} \neq j_{2}$ then the cardinality of

$$
A_{i_{1} i_{2} j_{1} j_{2}}=P_{i_{1} * j_{1}} \cap P_{i_{2} * j_{1}} \cap P_{i_{1} * j_{2}} \cap P_{i_{2} * j_{2}},
$$

is at most one. Now since $A_{i_{1} i_{2} j_{1} j_{2}}$ is an intersection of four planes, it is clear that it is either a plane, a line, a point, or empty. We need only exclude the first two possibilities.

Since $i_{1} \neq i_{2}$, it must be that

$$
P_{i_{1} * j_{1}} \cap P_{i_{2} * j_{1}}=L_{j_{1}},
$$

is either empty or a line since it is the intersection of two different planes. Thus we may assume it is a line. Similarly, we may assume that

$$
P_{i_{1} * j_{2}} \cap P_{i_{2} * j_{2}}=L_{j_{2}},
$$

is a line.

Now (2) is exactly $L_{j_{1}} \cap L_{j_{2}}$. Thus to show it has cardinality at most 1 , we need only show that $L_{j_{1}}$ and $L_{j_{2}}$ are not the same line. Let us suppose that they are. We call the line $L$.

Let $K_{1}$ be the set of all $i$ so that $P_{i * j_{1}}$ contains the line $L$. The set of $\alpha$ so that $P_{\alpha}$ contains $L$ is identified to a line $K$ in $F_{p}^{3} \backslash\{(0,0,0)\}$. Thus

$$
K_{1}=M_{j_{1}} K
$$

is also a line where $M_{j_{1}}$ is the linear operator on $F_{p}^{3}$ induced by multiplication in $F_{p^{3}}$ by $j_{1}$. Similarly, we define $K_{2}$ to be the set of all $i$ so that $P_{i * j_{2}}$ contains the line $L$ and

$$
K_{2}=M_{j_{2}} K
$$

By our assumption $i_{1}, i_{2} \in K_{1}$ and $i_{1}, i_{2} \in K_{2}$. Since $K_{1}$ and $K_{2}$ are lines, this means

$$
K_{1}=K_{2}
$$

But

$$
K_{1}=M_{j_{1}} M_{j_{2}^{-1}} K_{2}=M_{j_{1} *\left(j_{2}\right)^{-1}} K_{2}=M_{j_{1} *\left(j_{2}\right)^{-1}} K_{1} .
$$

Thus the linear map $M_{j_{1} *\left(j_{2}\right)^{-1}}$ preserves the line $K_{1}$.

Now since $j_{1} \neq j_{2}$ it must be that $j_{1} *\left(j_{2}\right)^{-1} \neq 1$. We claim that any $M_{\alpha}$ with $\alpha \in F_{p^{3}} \backslash\{0,1\}$ cannot preserve any lines not containing the origin. This is a contradiction. Thus to prove that $I$ satisfies the (E)-condition, we need only prove the claim.

We proceed to prove the claim. There are two cases, $\alpha$ is real or $\alpha$ is complex. If $\alpha$ is real, then $\alpha=d$, where $0,1 \neq d \in F_{p}$. Then $M_{\alpha}$ takes the line

$$
a x+b y+c z=1,
$$


into

$$
a x+b y+c z=d .
$$

These are clearly different lines.

Now suppose that $\alpha$ is complex and that $M_{\alpha}$ preserves the line $K_{1}$. Then $M_{\alpha}$ preserves the two dimensional subspace of $F_{p}^{3}$ spanned by $K_{1}$. Thus the characteristic polynomial of $M_{\alpha}$ factors nontrivially over $F_{p}$. But this is impossible since the characteristic polynomial of $M_{\alpha}$ is an irreducible cubic over $F_{p}$ of which $\alpha$ is a root. (This is true since the representation $\alpha \longrightarrow M_{\alpha}$ is faithful so that $\alpha$ must be a root of the characteristic polynomial of $M_{\alpha}$. But $\alpha$ is a nonscalar element of $F_{p^{3}}$ which has no proper subfields larger than $F_{p}$.) Thus we have proven the claim and the theorem.

\section{References}

[CCW] A. Carbery, M. Christ, and J. Wright, Multidimensional van der Corput and sublevel set estimates, J. Amer. Math. Soc. 12 (1999), 981-1015.

[E] P. Erdös, On extremal problems of graphs and generalized graphs, Israel J. Math. 2 (1964), 183-190.

[GRS] D. Gunderson, V. Rodl, and A. Sidorenko, Extremal problems for sets forming Boolean algebras and complete partite hypergraphs, J. Combin. Theory Ser. A 88 (1999), 342367.

Department of Mathematics, Washington University, St. Louis, MO 63130, U.S.A.

E-mail address: nets@math.wustl.edu

Department of Mathematics, Washington University, St. Louis, MO 63130, U.S.A.

E-mail address: krop@math.wustl.edu

Department of Mathematics, Yale University, New Haven, CT 06520, U.S.A.

E-mail address: Mauro.maggioni@yale.edu 